\title{
On the Source of Innovation and the Art of Discovery in Physics
}

International Journal of Theoretical \& Computational Physics Review Article

\author{
Yu Anyang \\ Submitted : March $13^{\text {th }} 2020$ \\ Accepted : March 23 2020 \\ Published : March 27 2020
}

Correspondence author

\author{
Yu Anyang \\ College of Science \\ Liaoning Shihua University \\ Fushun \\ China - 113001
}

\begin{abstract}
There are many breakthroughs in the history of physics, which dates from putting forward scientific problems and contributes the progresses of science and technology eventually. The scientists in physics have the spirits of making innovation and overcoming difficulties. Moreover, they have also demonstrate the art of scientific discovery to the world.

Summary : Many breakthrough results in the history of physics originate from the raising of scientific questions and promote the true progress of science and technology. Physicists not only have the courage to innovate and face difficulties, but also can use the correct research methods to show the art of scientific discovery to the world.
\end{abstract}

Keywords: Asking Scientific Questions, The Art of Discovery in Physics, Watching For Unexpected Events, Thinking Conversion

Introduction

A scientific question refers to a problem or contradiction raised in the course of scientific exploration that has not been solved or has no answer (explanation). It is usually associated with unrecognized natural phenomena or scientific mysteries. The great scientist Einstein once said, "It is often more important to ask a question than to solve it." Asking new questions, new possibilities, and looking at old questions from a new perspective requires creativity and imagination, and ultimately, it promotes real progress in science.

A series of breakthrough achievements in the history of physics can not only give us knowledge, but also give us inspiration. Physicists' courage to innovate, and the spirit of overcoming difficulties certainly admires us, but at the same time, we should also see the innovative thinking of physicists and the methods and arts in scientific research.

\section{The problem is the source of innovation in physics}

The process of physics discovery is also the process of asking questions and solving problems. In the process of discovering new concepts and laws of physics, raising scientific questions is a crucial link. It can be said that the problem is the source of innovation in physics.

Physics discoveries benefit from capturing scientific problems.

Finding contradictions and asking questions is the starting point of scientific research. In the development of physics, many physics concepts have emerged, and many laws of physics have been discovered. All of this comes from people asking scientific question [1]. After a series of achievements in classical mechanics, thermodynamics, statistical mechanics, and electrodynamics, many people believe that the grand

scientific building of physics has been completed, and it seems that the younger physicists need only do some patchy repair work. However, two new problems that plagued the physics community at the time appeared, one from the MichelsonMorley experiment and the other related to blackbody radiation. At that time, some people said that these two problems were two dark clouds appearing in the clear sky of physics. However, it is these two dark clouds that set off a profound revolution in the history of physics; the first problem led to the creation of relativity; the second problem led to the birth of quantum mechanics. In 1900, Plank introduced the famous hypothesis of quantization of energy to explain the problems encountered by black body radiation in classical physics, successfully explained the experimental facts of black body radiation, and opened a new era of quantum theory [2]. In the history of physics, such examples are endless. In 1905, Einstein proposed the photo-quantum theory in light of the contradiction between the experimental facts of the photoelectric effect and the classical concepts, and perfectly explained the experimental fact of the photoelectric effect [3]. Numerous examples reflect the crucial role of problems in the development of physics.

On the other hand, in the process of physicist's exploration, new problems will be induced with the solution of the original scientific problems; and the solution of new problems will lead to the subsequent questions. This chain of interlocking problems has promoted the spiral rise of physics and has continued to develop and progress [4]. After Zeman discovered the problem that the spectral line was split in the magnetic field in 1896, the famous Lorentz gave a correct theoretical explanation for this phenomenon, and later called it the normal Zeman effect. The old problems were solved, and new problems appeared. In December 1897, Preston reported that

$\overline{\text { I J T C Physcics } 2020 \quad \text { www.unisciencepub.com Volume1| Issue } 1}$ 
in many experimental cases, the number of divisions may not be three, and the intervals are not the same. This phenomenon is called the "abnormal" Zeeman effect. Although many people tried it, none of them could be reasonably explained. Thirty years later, Dutch scholars Uhlenbeck and Goodzmit proposed the electron spin hypothesis, which solved the problem of anomalous Zeeman effect [5].

On the surface, many physics discoveries are accidental. Some people seized the opportunity and fortunately observed the laws of physics. But in fact, anyone actively explores according to certain problems, and finally makes major discoveries. As we all know, light can show both the characteristics of waves and the characteristics of particles; it has wave-particle duality. However, in the early 20th century, when some physicists were puzzled by the wave-particle image of light, a French young man De Broglie, who had just shifted from the study of history to the study of physics, turned the wave of light The particle duality is extended to all material particles and has made a landmark contribution in the history of physics. De Broglie's success is not accidental, because people who study history like to think about problems by analogy. His theoretical basis is: Since photons have wave-particle duality, do other microscopic particles have such characteristics. Moving along this line of thought can indeed take a revolutionary step towards the creation of quantum mechanics [6]

Asking questions and solving problems is an excellent quality of a physicist.

Scientists' innovation is reflected in their ability to ask scientific questions. In 1930, when physicist Bot bombarded beryllium with alpha particles, he found that beryllium radiated a very penetrating ray, which would not deflect in electric and magnetic fields, and penetrated $2 \mathrm{~cm}$. The strength of the thick lead is only reduced by $13 \%$. At that time, the academic community believed that, of all the rays produced by the bombarded matter, only gamma rays could penetrate thick lead plates. However, Chadwick raised a very valuable scientific question. If this unknown neutral particle is a gamma photon, its energy is much greater than the energy of this unknown neutral particle. Therefore, such unknown neutral particles cannot be gamma rays. So what exactly is it? Chadwick finally discovered neutrons through careful experimental research [7]. With the raising of scientific questions, people can search and eventually produce innovative results. Being good at asking questions and solving problems is, to a large extent, the embodiment of outstanding scientists' creative thinking and creative talents. In 1827, botanist Brown first observed that pollen particles continued to move irregularly. At the time, people did not understand the cause of this phenomenon and called it the Brownian mystery. Scientists have studied this scientific problem for 50 years, and it was not until 1877 that Delso correctly pointed out that Brownian motion was caused by the imbalance of particles in the medium. In the early 20th century, Einstein and others' theoretical and experimental research on the Brownian motion gave Brownian motion a very clear explanation [8].
In short, asking scientific questions is the beginning of physics innovation. Only by being good at asking scientific questions can there be real scientific innovation.

\section{Discovery art of physics}

In the development of physics, there are countless historical discoveries. These discoveries embody the joy of physicists' success and the pain of failure, and they also include their research methods and art of exploring the mysteries of nature [9].The art of discovery by physicists is manifested in the following areas: Pay attention to details, see big in small.

Physicists sometimes encounter results that differ slightly from expected results in their scientific research. If you can keep on paying attention to these details, you will often lead to unexpected and unexpected discoveries. The so-called "details determine success or failure." Physicists can do this with keen insight and rigorous scientific attitude. As early as 1890, the British physics professor Schuster had studied the deflection of cathode rays in hydrogen discharge tubes, and calculated that the charge-to-mass ratio of the particles constituting the cathode ray was more than a thousand times the chargeto-mass ratio of hydrogen ions. But he couldn't believe his measurements. Because he believes that the result of a cathode ray particle having a mass of less than one thousandth of a hydrogen atom is ridiculous. In 1897, a German scholar Kaufman did a similar experiment, but he dare not admit that the cathode rays are particles. Thomson bravely proposed that there were particles much smaller than the atom. He confirmed through a series of simple and clever experiments that the cathode ray is composed of electrons. For the first time, humans have experimentally demonstrated the existence of a basic particle-electron. Thomson's experiment, grasping the details of previous experiments, showed that the small and the big, not only proved the existence of the electron, but also measured the charge-mass ratio of the electron, which caused a great sensation in the physics community at that time [10].

\section{Breaking the Routine}

Good physicists are good at digging out unusual truths in ordinary events that people are accustomed to. See the true meaning of the problem in unexpected places. This kind of curiosity caused by ordinary people to turn a blind eye and feel bland is often leading to major discoveries. The so-called "seemingly the most amazing." Newton is a well-known British scientist. When he was young, he often liked to stare at the stars at night. When the moon is clear and the stars are flickering, many questions often twitch in his mind: Why does the moon in the sky and the stars, large and small, always hang in the sky and never fall? Once he was reading under an apple tree, and suddenly, a ripe apple fell from the tree. He suddenly asked: Why doesn't Apple float in the air, but will it land vertically? Does the earth have an attraction for it? Newton conducted indepth research on life phenomena such as apple landing. Later, he discovered that there is mutual attraction between all objects in the universe, that is, gravity. In addition, Newton explained the tidal phenomenon caused by the gravitation of the moon and the sun. In 1687, Newton published the law of gravity in The Mathematical Principles of Natural Philosophy. It not only 
reveals the laws of the movement of objects on the earth, but also the laws of the movement of celestial bodies, which has a profound impact on the development of physics and astronomy [11]. The phenomenon of Apple landing is commonplace, and there are similar phenomena everywhere in life, but Newton was able to think about such trivial things that everyone thinks are ordinary, and finally discovered the important natural law that promotes human progress-the law of universal gravity. Deserves to be an outstanding physicist.

\section{Unexpected, Reasonable}

In the course of experiments looking for expected results, scientists will also pay attention to observe other things generated by these experiments and analyze these unexpected results. If these new results deviate from the original assumptions or expected results, they often give birth to new scientific discoveries and herald the birth of a new milestone in physics. Oster is a Danish physicist. He once said: I don't like the boring lectures without experiments. All scientific research starts from experiments. In April 1820, he gave a demonstration experiment to the students in the classroom. When he moved a live wire over the small magnetic needle, he found that the magnetic needle suddenly moved. This phenomenon did not attract the attention of the audience, and this unexpected phenomenon did not escape Oster's eyes. He has carefully studied the magnetic effect of this current, and has performed many experiments. It was found that the magnetic needle deflects around the current. Above the wire and below the wire, the magnetic needle deflects in the opposite direction [12]. Oster discovered that the magnetic phenomenon of electric current seems to be accidental, but it is not; because he has always believed that there must be some connection between electricity and magnetism, and electricity must be converted into magnetism. This is related to the fact that he was deeply influenced by Kant's philosophical thoughts when he was in college, thinking that all natural forces come from the same source and can be transformed into each other. Oster's story tells us that when truth hits the tip of the nose, if you observe carefully and think correctly, you can still get the truth. Opportunities belong to those who are prepared. "Watch out for accidents" is the motto of scientific researchers. It is reasonable that some "unexpected" incidents in scientific research can be captured by scientists and analyzed in depth. Because scientists have been focusing on this issue for a long time, and are prepared to have the foundation and conditions for new breakthroughs. We say that opportunities favor only prepared minds. Having this "mind" is not easy, and often requires sufficient knowledge accumulation to focus on scientific research for a long time. The so-called "as easy as it is the most difficult." Madame Curie is famous all over the world. She pioneered the theory of radioactivity, invented the technology of separating radioisotopes, and also discovered two new elements, thorium and radium, and was the first person on earth to win the Nobel Prize twice. In the years when Mrs. Curie was studying the separation and purification of metallic radium, she had to sleep and forget to eat. Not only did she suffer the loss of her husband, but she also developed leukemia due to exposure to excessive radioactive material [13].

\section{Change between thinking}

Movement and static are opposites. The two are relative. In scientific research, sometimes it is necessary to take static while moving, and sometimes it is necessary to turn static into action. There are many examples of this change of thinking between dynamic and static. As is known to all, synchrotron radiation is electromagnetic radiation emitted by relativistic charged particles as they travel along curved orbits under the influence of electromagnetic fields. Originally, it was observed on a synchrotron, so it was called synchrotron radiation, or synchrotron for short. For a long time, synchrotron radiation is a "by-product" not welcomed by high-energy physicists, because it consumes the energy of the accelerator and hinders the increase of particle energy. However, scientists turned waste into treasure, using synchrotron radiation sources to carry out cutting-edge scientific research that other light sources cannot perform. This is because the synchrotron radiation has a continuous spectrum from the far infrared to the X-ray range, or it is a high-quality pulsed light source with high intensity, high collimation, high polarization, and precise and controllable characteristics. In today's era, synchrotron radiation sources have already become a state-of-the-art, irreplaceable tool for basic and applied research in the fields of life sciences, materials sciences, environmental sciences, physics, chemistry, medicine, geology and other disciplines. The electronics industry, the pharmaceutical industry, the petroleum industry, the chemical industry, the biological engineering, and the micro-processing industry have important and widespread applications [14]. The above facts reflect the idea that physicists are good at changing between static and dynamic, turning waste into treasure, that is, engaging in one extreme of things to the other extreme of things to deal with problems.

When two cars are driving in the same direction and at the same speed, the people in the car see the people in the car next to them in a relatively static state, while the two look at the people on the roadside, and they are in a relative motion state. All these have revealed to us that in the process of material movement and development, conditions are an important factor that cannot be ignored.

\section{Conclusion}

The problem is the source of innovation in physics. The process of physics discovery is also the process of asking questions and solving problems. Asking questions and solving problems is an excellent quality of a physicist. Anyone actively explores according to certain problems, and finally makes major discoveries in physics, which promotes the continuous development of physics.

The artistic findings of physicists in scientific research are expressed in the following aspects:

1. With keen insight and rigorous scientific attitude. Understand that "details determine success or failure."

2. Good at digging out unusual truths in ordinary events that people are accustomed to. 
3. Opportunities belong to people who are prepared. Good physicists can not only seize opportunities, but also sometimes create opportunities and break through innovation.

4. In scientific research, pay attention to thinking conversion; be able to take static while moving, and sometimes turn static into action.

\section{References}

1. Xiang Yihe (1994) "The Origin of Basic Concepts and Laws of Physics". Higher Education Press 1994.

2. Cayori, Dai Nianzu (2002) "History of Physics". Guangxi Normal University Press, 2002.

3. Xu Liangying, et al. (1983)"Einstein Anthology" (Volume 1). Beijing Commercial Press, 1983.

4. Xie Lizhong (1982) "Philosophy of Science" Taipei Liming Cultural Enterprise, 1982.

5. Yang Fujia's (2000) Atomic Physics (Third Edition). Higher Education Press, 2000.

6. Zeng Jinyan"Introduction to Quantum Mechanics"

7. Garmov (1981) History of Physics. Beijing Commercial Press, 1981.

8. Einstein (1962) Infeld's "The Evolution of Physics". Shanghai Science and Technology Press, 1962.

9. Cheng Jiubiao (2003)"The Art of Physics Discovery". China Ocean University Press, 2003.

10. Yan Zhenyu (2002) A New Edition of History of Physics. Guizhou Science and Technology Press, 2002.

11. Wolfe (1997) History of Science, Technology and Philosophy in the 16th and 17th Century. Beijing Commercial Press, 1997.

12. Chen Binggan (2001)"Special Research on Electromagnetics". Higher Education Press, 2001.

13. Guang Zhong (1988)"History of Physics". Beijing Qiushi Press, 1988.

14. Ni Guangjiong (1999) Physics Changing the World (Second Edition). Fudan University Press, 1999.

Copyright: (C2020 Yu Anyang. This is an open-access article distributed under the terms of the Creative Commons Attribution License, which permits unrestricted use, distribution, and reproduction in any medium, provided the original author and source are credited. 